\title{
A NEW APPROACH TO NORM INEQUALITIES ON WEIGHTED AND VARIABLE HARDY SPACES
}

\author{
David Cruz-Uribe, OFS, Kabe Moen and Hanh Van Nguyen \\ University of Alabama, Department of Mathematics \\ Tuscaloosa, AL 35487, U.S.A.; dcruzuribe@ua.edu \\ University of Alabama, Department of Mathematics \\ Tuscaloosa, AL 35487, U.S.A.; kabe.moen@ua.edu \\ University of Alabama, Department of Mathematics \\ Tuscaloosa, AL 35487, U.S.A.; hanhnguyenvan@gmail.com
}

\begin{abstract}
We give new proofs of Hardy space estimates for fractional and singular integral operators on weighted and variable exponent Hardy spaces. Our proofs consist of several interlocking ideas: finite atomic decompositions in terms of $L^{\infty}$ atoms, vector-valued inequalities for maximal and other operators, and Rubio de Francia extrapolation. Many of these estimates are not new, but we give new and substantially simpler proofs, which in turn significantly simplifies the proofs of the Hardy spaces inequalities.
\end{abstract}

\section{Introduction}

In this paper we give new proofs of norm inequalities for Calderón-Zygmund singular integrals and fractional integral operators on the weighted Hardy spaces, $H^{p}(w)$, and the variable Hardy spaces, $H^{p(\cdot)}$. The theory of weighted Hardy spaces is classical: see the monograph by Strömberg and Torchinsky [38] and the earlier paper by García-Cuerva [15]. Variable Hardy spaces are Hardy spaces defined in the scale of the variable Lebesgue spaces $L^{p(\cdot)}$, a generalization of the $L^{p}$ spaces that has been an active area of research for the past two decades: see the books [5, 12]. The variable Hardy spaces were introduced more recently: see [11, 31]. (Complete definitions of these spaces will be given in Section 2 below.)

We give Hardy space estimates for three types of operators: singular integrals of convolution type, fractional integral operators (which are also convolution operators), and singular integrals of non-convolution type. A Calderón-Zygmund singular integral of convolution type is an operator $T$ such that for all $f \in C_{c}^{\infty}$,

$$
T f(x)=\text { p.v. } \int_{\mathbf{R}^{n}} K(x-y) f(y) d y,
$$

where the kernel $K$ is defined on $\mathbf{R}^{n} \backslash\{0\}$ and has regularity of order $N+1$ :

$$
\left|\partial_{x}^{\alpha} K(x)\right| \leq \frac{A_{\alpha}}{|x|^{n+|\alpha|}}
$$

https://doi.org/10.5186/aasfm.2020.4526

2010 Mathematics Subject Classification: Primary 42B20, 42B25, 42B30, 42B35.

Key words: Weighted Hardy spaces, variable Hardy spaces, extrapolation, singular integrals, fractional integrals.

The first author is supported by research funds from the Dean of the College of Arts \& Sciences, the University of Alabama, and the second author is supported by the Simons Foundation. 
for all $\alpha$ such that $|\alpha| \leq N+1$, where $N$ is a sufficiently large integer. For $0<p \leq 1$, if $N>\left\lfloor n\left(\frac{1}{p}-1\right)\right\rfloor$, then $T: H^{p} \rightarrow H^{p}$. (See Stein [37] or García-Cuerva and Rubio de Francia [16].)

Our first two theorems extend this result to weighted and variable exponent Hardy spaces; again, for brevity we defer some technical definitions to Section 2. For a weight $w \in A_{\infty}$ let $r_{w}=\inf \left\{p: w \in A_{p}\right\}$.

Theorem 1.1. Given a weight $w \in A_{\infty}$ and $0<p<\infty$, suppose that $T$ is a Calderón-Zygmund singular integral operator of convolution type with regularity of order $N+1$, where

$$
N>\left\lfloor n\left(\frac{r_{w}}{p}-1\right)\right\rfloor
$$

Then $T: H^{p}(w) \rightarrow H^{p}(w)$.

Remark 1.2. Weighted Hardy space estimates for singular integrals (actually for the more general class of multipliers) were proved by Strömberg and Torchinsky [38]. Theorem 1.1 was proved by $\mathrm{Lu}$ and $\mathrm{Zhu}$ [27] for singular integrals with $C^{\infty}$ kernels; see the bibliography of their paper for earlier results.

Theorem 1.3. Given an exponent function $p(\cdot) \in \mathcal{P}_{0}$, suppose $0<p_{-} \leq p_{+}<\infty$ and $p(\cdot) \in L H$. Suppose further that $T$ is a Calderón-Zygmund singular integral operator of convolution type with regularity of order $N+1$, where

$$
N>\left\lfloor n\left(\frac{1}{p_{-}}-1\right)\right\rfloor .
$$

Then $T: H^{p(\cdot)} \rightarrow H^{p(\cdot)}$.

Remark 1.4. Theorem 1.3 was first proved independently in $[11,31]$.

Second, we consider the fractional integral operator, $I_{\alpha}$. Given $0<\alpha<n$, define

$$
I_{\alpha} f(x)=\int_{\mathbf{R}^{n}} \frac{f(y)}{|x-y|^{n-\alpha}} d y .
$$

If $0<p<\frac{n}{\alpha}$ and $\frac{1}{p}-\frac{1}{q}=\frac{\alpha}{n}$, then $I_{\alpha}: H^{p} \rightarrow H^{q}$. (See Stein [37] or Krantz [25].)

Theorem 1.5. Given $0<\alpha<n, 0<p<\frac{n}{\alpha}$, define $q$ by $\frac{1}{p}-\frac{1}{q}=\frac{\alpha}{n}$. If a weight $w$ is such that $w^{p} \in R H_{\frac{q}{p}}$, then $I_{\alpha}: H^{p}\left(w^{p}\right) \rightarrow H^{q}\left(w^{q}\right)$.

Remark 1.6. Theorem 1.5 was first proved by Strömberg and Wheeden [39]; see also Gatto, Gutiérrez and Wheeden [17].

Theorem 1.7. Given $0<\alpha<n$, and $p(\cdot) \in \mathcal{P}_{0}$, suppose $0<p_{-} \leq p_{+}<\frac{n}{\alpha}$ and $p(\cdot) \in L H$. Define $q(\cdot)$ by $\frac{1}{p(\cdot)}-\frac{1}{q(\cdot)}=\frac{\alpha}{n}$. Then $I_{\alpha}: H^{p(\cdot)} \rightarrow H^{q(\cdot)}$.

Remark 1.8. Theorem 1.7 was first proved by Rocha and Urciuolo [32]. See also Sawano [34, Theorem 5.1].

Third, we consider Calderón-Zygmund operators of non-convolution type, as defined by Coifman and Meyer [4]. An operator $T$ is a Calderón-Zygmund operator if it is a bounded operator on $L^{2}\left(\mathbf{R}^{n}\right)$, and if all $f \in L_{c}^{\infty}$ and $x \notin \operatorname{supp}(f)$,

$$
T f(x)=\int_{\mathbf{R}^{n}} K(x, y) f(y) d y
$$


where the distributional kernel coincides with a function $K$ defined away from the diagonal on $\mathbf{R}^{2 n}$ and satisfies the standard estimates

$$
\begin{gathered}
|K(x, y)| \leq \frac{C}{|x-y|^{n}}, \quad x \neq y \\
|K(x, y+h)-K(x, y)|+|K(x+h, y)-K(x, y)| \leq \frac{C|h|^{\delta}}{|x-y|^{n+\delta}}
\end{gathered}
$$

for all $|h| \leq \frac{1}{2}|x-y|$, where $C>0$ and $0<\delta \leq 1$.

For boundedness on Hardy spaces we will also need to assume two additional conditions on $T$. First, we will need that the kernel $K$ satisfies for some $N>0$ the additional smoothness condition

$$
\left|\partial_{y}^{\beta} K(x, y+h)-\partial_{y}^{\beta} K(x, y)\right| \leq \frac{C|h|^{\delta}}{|x-y|^{n+N+\delta}}
$$

for all $x \neq y,|h| \leq \frac{|x-y|}{2}$ and all $|\beta|=N$. Second, we will need that the operator $T$ has $L$ vanishing moments, in the sense that

$$
\int x^{\beta} T a(x) d x=0
$$

for all $(L+1, \infty)$ atoms $a$ and $|\beta| \leq L$. We note that this moment condition is satisfied by all convolution type singular integrals (see [21, Lemma 2.1]), and is a necessary condition for $T$ to map into unweighted $H^{p}$ (see [18, Theorem 7]).

Theorem 1.9. Given $w \in A_{\infty}$ and $0<p<\infty$, suppose that $T$ is a CalderónZygmund operator associated with a kernel $K$ that satisfies (1.3) for all $|\beta|=L+1$, and suppose $T$ has $L$ vanishing moments (1.4), where

$$
L=\max \left(\left\lfloor n\left(\frac{r_{w}}{p}-1\right)\right\rfloor,-1\right) .
$$

(If $L=-1$, then condition (1.4) can be omitted.) Then $T: H^{p}(w) \rightarrow H^{p}(w)$.

Remark 1.10. Norm inequalities for non-convolution Calderón-Zygmund operators have been considered by a number of authors. In the unweighted case, Alvarez and Milman [1] show that if $T$ is a Calderón-Zygmund operator, then $T: H^{p} \rightarrow H^{p}$ for $\frac{n}{n+\delta}<p \leq 1$, where $\delta$ is the exponent in (1.3). From Theorem 1.9 we get the slightly larger range $\frac{n}{n+1}<p \leq 1$ but only if we assume that $L=0$, so that we require greater regularity on the operator $T$ than they do.

Their results were generalized to the full range of $0<p \leq 1$ in the unweighted case by Hart and $\mathrm{Lu}$ [22], and to $0<p<\infty$ and $w \in A_{\infty}$ in the weighted case by Hart and Oliveira [23]. Their results are not directly comparable to ours, though the conditions on the weights and the range of $p$ are roughly the same. They assume a great deal more regularity on the kernel $K$ : they require that a version of (1.3) holds that involves derivatives in both $x$ and $y$, whereas we only require derivatives in $y$. We also note that rather than (1.4), they require that $T^{*}\left(x^{\beta}\right)=0$. The two are formally equivalent, but the latter requires additional machinery to define.

Theorem 1.11. Given an exponent function $p(\cdot) \in \mathcal{P}_{0}$, suppose $0<p_{-} \leq$ $p_{+}<\infty$ and $p(\cdot) \in L H$. Suppose further that $T$ is a Calderón-Zygmund operator associated with a kernel $K$ that satisfies (1.3) for all $|\beta|=L+1$, and suppose $T$ has 
$L$ vanishing moments (1.4), where

$$
L=\max \left(\left\lfloor n\left(\frac{1}{p_{-}}-1\right)\right\rfloor,-1\right) .
$$

(If $L=-1$, then condition (1.4) can be omitted.) Then $T: H^{p(\cdot)} \rightarrow H^{p(\cdot)}$.

Remark 1.12. Theorem 1.11 is new. However, a slightly weaker result was implicit as a special case of a result for multilinear Calderón-Zygmund operators recently proved in [10].

Most of our results are not new; however, our main contribution in this paper is our new approach to the proofs, which we believe is significantly simpler and more transparent than existing proofs. Therefore, before giving the actual proofs, we want to summarize their main ideas.

The first component is a finite atomic decomposition in terms of $L^{\infty}$ atoms. In the unweighted case such a decomposition was first proved by Meda, Sjögren and Vallarino [29]. They showed that on a dense set in $H^{p}, 0<p<1$, it is possible to write a function $f$ as a finite sum of $(N, \infty)$ atoms

$$
f=\sum_{i=1}^{M} \lambda_{i} a_{i}
$$

in such a way that

$$
\|f\|_{H^{p}} \approx\left(\sum_{i=1}^{M} \lambda_{i}^{p}\right)^{\frac{1}{p}} .
$$

The advantage of such a decomposition is that it allows the interchange of the operator and the sum without having to worry about the convergence of the sum, and reduces the problem to estimating the operator on individual atoms. In a previous paper [10] we extended the finite atomic decomposition to weighted Hardy spaces; here we prove it for variable Hardy spaces, generalizing a result proved in [11].

The second component of our proofs starts with a vector-valued inequality due to Grafakos and Kalton [19]: given any collection of cubes $Q_{k}$ and functions $g_{k}$ with $\operatorname{supp}\left(g_{k}\right) \subset Q_{k}$, then for $0<p \leq 1$,

$$
\left\|\sum_{k} g_{k}\right\|_{p} \lesssim\left\|\sum_{k}\left(f_{Q_{k}} g_{k} d x\right) \chi_{Q_{k}}\right\|_{p} .
$$

Their proof was quite technical, since the cubes are not assumed to be disjoint. Though not needed, we give a very elementary proof of this inequality of Grafakos and Kalton. More importantly, however, we prove versions that hold for $p>1$ on weighted spaces, and on variable exponent spaces. Using these, we can divide the estimate of the operator on individual atoms into their local and global pieces and then estimate the local piece using unweighted $L^{q}$ estimates for $q>\max (p, 1)$. We also give a new proof of a variant of the Grafakos-Kalton lemma that is used in the off-diagonal case for the fractional integral operator and that in the weighted case is due to Strömberg and Wheeden [39] and in the variable exponent case to Sawano [34]. Again our proofs are much simpler than the original ones.

The third component of our proofs are vector-valued inequalities for the HardyLittlewood maximal operator. It is a classical result due to Fefferman and Stein [14] 
that for $1<p, r<\infty$,

$$
\left\|\left(\sum_{k}\left(M g_{k}\right)^{r}\right)^{\frac{1}{r}}\right\|_{p} \lesssim\left\|\left(\sum_{k}\left|g_{k}\right|^{r}\right)^{\frac{1}{r}}\right\|_{p} .
$$

A similar inequality also holds for the fractional maximal operator, $M_{\alpha}, 0<\alpha<n$, and is due to Ruiz and Torrea [33]: if $1<p<\frac{n}{\alpha}, \frac{1}{p}-\frac{1}{q}=\frac{\alpha}{n}$, and $1<r<\infty$,

$$
\left\|\left(\sum_{k}\left(M_{\alpha} g_{k}\right)^{r}\right)^{\frac{1}{r}}\right\|_{q} \lesssim\left\|\left(\sum_{k}\left|g_{k}\right|^{r}\right)^{\frac{1}{r}}\right\|_{p} .
$$

Both of these inequalities extend to weighted and variable Lebesgue spaces, and we use them to estimate the global part of the operator applied to an atom.

The final component of our proofs is the theory of Rubio de Francia extrapolation. Using the reformulation in terms of extrapolation pairs [9], the above vector-valued inequalities for maximal operators are an immediate consequence of the corresponding scalar inequalities. The variants of the Grafakos-Kalton lemma described above also follow easily from extrapolation. For these we need to use more recent versions of extrapolation, including limited range extrapolation [8], extrapolation with respect to reverse Hölder weights [3], and extrapolation into variable Lebesgue spaces [5, 6, 9]. Our proofs also depend on several new extrapolation results which we prove here.

The remainder of this paper is organized as follows. In Section 2 we give the necessary definitions and results about weighted and variable exponent Hardy spaces. In Section 3 we give the versions of extrapolation that we use and prove the new versions we need. In Section 4 we state and prove the vector-valued inequalities we use. In Section 5 we prove Theorems 1.1 and 1.3; in Section 6 we prove Theorems 1.5 and 1.7; and in Section 7 we prove Theorems 1.9 and 1.11. Even though the proofs are given in separate sections, we want to emphasize that they all have a common structure. Finally, in Section 8 we briefly discuss generalizing our results to Hardy spaces defined with respect to other scales of Banach function spaces.

Throughout this paper, $n$ will denote the dimension of the underlying space, $\mathbf{R}^{n}$. By a cube $Q$ we will always mean a cube whose sides are parallel to the coordinate axes, and for $\tau>0, \tau Q$ will denote the cube with the same center such that $\ell(\tau Q)=$ $\tau \ell(Q)$. Given $Q$, let $Q^{*}=2 \sqrt{n} Q$ and $Q^{* *}=\left(Q^{*}\right)^{*}$. We define the average of a function $f$ on $Q$ by $f_{Q} f d x=|Q|^{-1} \int_{Q} f d x$. By $C$, $c$, etc. we will mean constants that may depend on the underlying parameters in the proof. Their values may change at each appearance. Given two quantities $A$ and $B$, we will write $A \lesssim B$ if there exists a constant $c$ such that $A \leq c B$. If $A \lesssim B$ and $B \lesssim A$, we write $\widetilde{A} \approx B$.

\section{Preliminaries}

In this section we gather together some basic results about weighted and variable exponent spaces. We begin with some information about weights. For more information, see $[9,13,16]$. By a weight we mean a non-negative, locally integrable function $w$ such that $0<w(x)<\infty$ a.e. For $1<p<\infty$, a weight is in the Muckenhoupt class $A_{p}$ if for every cube $Q$,

$$
f_{Q} w d x\left(f_{Q} w^{1-p^{\prime}} d x\right)^{p-1} \leq C,
$$


and when $p=1, w \in A_{1}$ if for every cube $Q$ and a.e. $x \in Q$,

$$
f_{Q} w d x \leq C w(x)
$$

If $1<p<\infty$ and $w \in A_{p}$, then the Hardy-Littlewood maximal operator, defined by

$$
M f(x)=\sup _{Q} f_{Q}|f(y)| d y \cdot \chi_{Q}(x),
$$

is bounded on $L^{p}(w)$.

Define the set

$$
A_{\infty}=\bigcup_{p \geq 1} A_{p}
$$

Given a weight $w \in A_{\infty}$, recall $r_{w}=\inf \left\{r \geq 1: w \in A_{r}\right\}$. A weight $w \in A_{\infty}$ if and only if $w \in R H_{s}$ for some $s>1$ : that is, for every cube $Q$,

$$
\left(f_{Q} w^{s} d x\right)^{\frac{1}{s}} \leq C f_{Q} w d x
$$

Furthermore, we have the property that $w \in R H_{s}$ if and only if $w^{s} \in A_{\infty}$. The limiting class $R H_{\infty}$ is defined to be all $w$ such that for every cube $Q$ and a.e. $x \in Q$,

$$
w(x) \leq C f_{Q} w d x .
$$

Given $1<p, q<\infty$, a weight satisfies the $A_{p, q}$ condition of Muckenhoupt and Wheeden if for every cube $Q$,

$$
\left(f_{Q} w^{q} d x\right)^{\frac{1}{q}}\left(f_{Q} w^{-p^{\prime}} d x\right)^{\frac{1}{p^{\prime}}} \leq C .
$$

It follows from the definition that $w \in A_{p, q}$ if and only if $w^{q} \in A_{1+\frac{q}{p^{p}}}$. When $p=1$ and $q>1$; we say that $w \in A_{1, q}$ if for every cube $Q$ and almost every $x \in Q$,

$$
f_{Q} w^{q} d x \leq C w(x)^{q} .
$$

This is clearly equivalent to $w^{q} \in A_{1}$. Given $0 \leq \alpha<n$ and $1<p<\frac{n}{\alpha}$, define $q$ by $\frac{1}{p}-\frac{1}{q}=\frac{\alpha}{n}$. If $w \in A_{p, q}$ then the fractional maximal operator,

$$
M_{\alpha} f(x)=\sup _{Q}|Q|^{\frac{\alpha}{n}} f_{Q}|f(y)| d y \cdot \chi_{Q}(x),
$$

is bounded from $L^{p}\left(w^{p}\right)$ to $L^{q}\left(w^{q}\right)$.

We now define the weighted Hardy spaces $H^{p}(w), 0<p<\infty$. For more information, see [38]; also see [35]. Let $\mathcal{S}$ denote the Schwartz class of smooth functions. Given a (large) integer $N_{0}$, define

$$
\mathcal{S}_{N_{0}}=\left\{\phi \in \mathcal{S}: \int_{\mathbf{R}^{n}}(1+|x|)^{N_{0}}\left(\sum_{|\beta| \leq N_{0}}\left|\partial^{\beta} \phi(x)\right|^{2}\right) d x \leq 1\right\} .
$$

Given $\phi \in \mathcal{S}_{N_{0}}$, define the radial maximal operator

$$
M_{\phi} f(x)=\sup _{t>0}\left|\phi_{t} * f(x)\right|,
$$


where $\phi_{t}(x)=t^{-n} \phi(x / t)$. Define the grand maximal operator

$$
\mathcal{M}_{N_{0}} f(x)=\sup _{\phi \in \mathcal{S}_{N_{0}}} M_{\phi} f(x) .
$$

Given $w \in A_{\infty}$ and $0<p<\infty$, define the weighted Hardy space to be the set of distributions

$$
H^{p}(w)=\left\{f \in \mathcal{S}^{\prime}: \mathcal{M}_{N_{0}} f \in L^{p}(w)\right\}
$$

with quasi-norm $\|f\|_{H^{p}(w)}=\left\|\mathcal{M}_{N_{0}} f\right\|_{L^{p}(w)}$. If $p>1$ and $w \in A_{p}$, then $H^{p}(w)=$ $L^{p}(w)$, since $M_{N_{0}} f$ is dominated pointwise by $M f$, where $M$ is the Hardy-Littlewood maximal operator. We can choose the value $N_{0}$, depending only on $n, p$ and $r_{w}$, so that $f \in H^{p}(w)$ if and only if for any $\phi \in \mathcal{S}_{N_{0}}, M_{\phi} f \in L^{p}(w)$. The value of $N_{0}$ chosen will be implicit in our constants below.

Given an integer $N>0$, we define an $(N, \infty)$ atom be a bounded function $a$ such that $\|a\|_{\infty} \leq 1, \operatorname{supp}(a) \subset Q$ for some cube $Q$, and such that for all $|\beta| \leq N$,

$$
\int_{\mathbf{R}^{n}} x^{\beta} a(x) d x=0
$$

Given $0<p<\infty$ and $w \in A_{\infty}$, let

$$
s_{w}=\left\lfloor N\left(\frac{r_{w}}{p}-1\right)\right\rfloor_{+} .
$$

If $N>s_{w}$, then any $(N, \infty)$ atom is in $H^{p}(w)$. Moreover, every element of $f$ can be decomposed as the sum of atoms: if $f \in H^{p}(w)$, then there exists a sequence of $(N, \infty)$ atoms $\left\{a_{i}\right\}$ with supports $\left\{Q_{i}\right\}$, and $\lambda_{i}>0$ such that

$$
f=\sum_{i} \lambda_{i} a_{i}
$$

and

Given $N>s_{w}$, define

$$
\|f\|_{H^{p}(w)} \approx\left\|\sum_{i} \lambda_{i} \chi_{Q_{i}}\right\|_{L^{p}(w)} .
$$

$$
\mathcal{O}_{N}=\left\{f \in C_{0}^{\infty}: \int_{\mathbf{R}^{n}} x^{\beta} f(x) d x=0,0 \leq|\beta| \leq N\right\} .
$$

Each element of $\mathcal{O}_{N}$ is a multiple of an $(N, \infty)$ atom, so it follows from the atomic decomposition that $\mathcal{O}_{N}$ is dense in $H^{p}(w)$. Moreover, we have the following finite atomic decomposition that was proved in [10].

Proposition 2.1. Given $0<p<\infty$ and $w \in A_{\infty}$, fix $N>s_{w}$. Then if $f \in \mathcal{O}_{N}$, there exists a finite sequence $\left\{a_{i}\right\}_{i=1}^{M}$ of $(N, \infty)$ atoms with supports $Q_{i}$, and a nonnegative sequence $\left\{\lambda_{i}\right\}_{i=1}^{M}$ such that $f=\sum_{i} \lambda_{i} a_{i}$ and

$$
\left\|\sum_{i=1}^{M} \lambda_{i} \chi_{Q_{i}}\right\|_{L^{p}(w)} \leq C\|f\|_{H^{p}(w)} .
$$

Remark 2.2. As an immediate consequence of Proposition 2.1 and the density of $\mathcal{O}_{N}$ in $H^{p}(w)$, to prove that an operator $T$ extends to a bounded operator from $H^{p}(w)$ to itself, it suffices to show that if $f$ is a finite sum of $(N, \infty)$ atoms, then

$$
\|T f\|_{L^{p}(w)} \lesssim\left\|\sum_{i=1}^{M} \lambda_{i} \chi_{Q_{i}}\right\|_{L^{p}(w)} .
$$


We now define the variable exponent Hardy spaces. We first state some basic results about variable Lebesgue spaces. For more information, see [5]. Let $\mathcal{P}_{0}$ be the collection of all measurable functions $p(\cdot): \mathbf{R}^{n} \rightarrow(0, \infty)$. Define

$$
p_{-}=\underset{x \in \mathbf{R}^{n}}{\operatorname{ess} \inf } p(x), \quad p_{+}=\underset{x \in \mathbf{R}^{n}}{\operatorname{ess} \sup } p(x) .
$$

An exponent $p(\cdot) \in \mathcal{P}_{0}$ is log-Hölder continuous, denoted by $p(\cdot) \in L H$, if

$$
|p(x)-p(y)| \leq \frac{C_{0}}{-\log (|x-y|)}, \quad|x-y|<\frac{1}{2},
$$

and if there exists constants $p_{\infty}$ and $C_{\infty}$ such that

$$
\left|p(x)-p_{\infty}\right| \leq \frac{C_{\infty}}{\log (e+|x|)}
$$

We define $L^{p(\cdot)}$ to be the set of all measurable functions $f$ such that

$$
\|f\|_{p(\cdot)}=\inf \left\{\lambda>0: \int_{\mathbf{R}^{n}}\left(\frac{|f(x)|}{\lambda}\right)^{p(x)} d x \leq 1\right\}<\infty .
$$

This defines a quasi-norm and $L^{p(\cdot)}$ is a quasi-Banach function space; if $p_{-} \geq 1$, it is a Banach space. If $p(\cdot) \in \mathcal{P}_{0}, p_{-}>1$, and $p(\cdot) \in L H$, then the Hardy-Littlewood maximal operator is bounded on $L^{p(\cdot)}$. If $1<p_{-} \leq p_{+} \leq \frac{n}{\alpha}$ and $q(\cdot)$ is defined by $\frac{1}{p(\cdot)}-\frac{1}{q(\cdot)}=\frac{\alpha}{n}$, then the fractional maximal operator $M_{\alpha}$ maps $L^{p(\cdot)}$ to $L^{q(\cdot)}$.

Given $p(\cdot) \in \mathcal{P}_{0}$, define the variable Hardy space $H^{p(\cdot)}$ to be the set of all distributions $f$ such that $\mathcal{M}_{N_{0}} f \in L^{p(\cdot)}$. Again, we may fix $N_{0}$ depending only $p(\cdot)$ and $n$ such that $f \in H^{p(\cdot)}$ if and only if $M_{\phi} f \in L^{p(\cdot)}$, where $\phi \in \mathcal{S}$. We have the following atomic decomposition (see [11]): if

$$
N>s_{p(\cdot)}=\left\lfloor n\left(\frac{1}{p_{-}}-1\right)\right\rfloor_{+},
$$

then every $f \in H^{p(\cdot)}$ can be written as the sum of $(N, \infty)$ atoms,

$$
f=\sum_{i} \lambda_{i} a_{i}
$$

and

$$
\|f\|_{H^{p(\cdot)}} \approx\left\|\sum_{i} \lambda_{i} \chi_{Q_{i}}\right\|_{L^{p(\cdot)}} .
$$

Remark 2.3. This is a slightly different formulation of the atomic decomposition than that given in [11]. However, the difference lies in the normalization of the atoms and is not significant for our purposes.

Similar to the weighted theory, if we fix $N>s_{p(\cdot)}$, then the atomic decomposition implies that $\mathcal{O}_{N}$ is dense in $H^{p(\cdot)}$. Furthermore, we have a finite atomic decomposition for elements of $\mathcal{O}_{N}$. The proof of the following result is nearly the same as the proof of Proposition 2.1; the proof can be adapted to the variable Lebesgue space setting using the ideas used to prove a similar finite atomic decomposition in [11, Theorem 7.8].

Proposition 2.4. Given $p(\cdot) \in \mathcal{P}_{0}$, suppose $0<p_{-} \leq p_{+}<\infty$ and $p(\cdot) \in L H$. Fix $0<p_{0}<p_{-}$. Then for any $N$ such that

$$
N>\left\lfloor N\left(\frac{1}{p_{0}}-1\right)\right\rfloor_{+},
$$


given any $f \in \mathcal{O}_{N}$, there exists a finite sequence $\left\{a_{i}\right\}_{i=1}^{M}$ of $(N, \infty)$ atoms with supports $Q_{i}$, and a non-negative sequence $\left\{\lambda_{i}\right\}_{i=1}^{M}$ such that $f=\sum_{i} \lambda_{i} a_{i}$ and

$$
\left\|\sum_{i=1}^{M} \lambda_{i} \chi_{Q_{i}}\right\|_{L^{p(\cdot)}} \lesssim\|f\|_{H^{p(\cdot)}} .
$$

Remark 2.5. Unless $N\left(p_{-}^{-1}-1\right)$ is an integer, the lower bound for $N$ in Proposition 2.4 is the same as $s_{p(\cdot)}$ defined above.

\section{Extrapolation results}

In this section we review the theory of Rubio de Francia extrapolation which is used to prove weighted norm inequalities in weighted and variable exponent spaces. We also prove two new variants that we will use in Section 4 below.

We begin by recalling the abstract notion of extrapolation pairs. For more information, see [9]. We define a family of extrapolation pairs to be a family $\mathcal{F}$ of pairs of non-negative, measurable functions $(f, g)$. Whenever we write an inequality of the form

$$
\|f\|_{X} \lesssim\|g\|_{Y}, \quad(f, g) \in \mathcal{F}
$$

where $\|\cdot\|_{X}$ and $\|\cdot\|_{Y}$ are norms in a Banach or quasi-Banach space, we mean that this inequality holds for every pair $(f, g)$ in $\mathcal{F}$ such that $\|f\|_{X}<\infty$, and the constant is independent of the pair $(f, g)$. (If $X=Y=L^{p}(w)$ for some $w \in A_{q}$, then the constant can only depend on the $A_{q}$ constant of $w$ and not on $w$ itself. A similar restriction is assumed for off-diagonal estimates.) The assumption that $\|f\|_{X}<\infty$ is only for technical reasons in the proof; it can often be ignored. Given any pair $(f, g)$, we can replace it by the pairs $\left(\min (f, N) \chi_{B(0, N)}, g\right)$; then by Fatou's lemma (which still holds in the setting of quasi-Banach function spaces) we have that $\left\|\min (f, N) \chi_{B(0, N)}\right\|_{X}<\infty$ and

$$
\lim _{N \rightarrow \infty}\left\|\min (f, N) \chi_{B(0, N)}\right\|_{X}=\|f\|_{X} .
$$

To apply extrapolation to prove a norm inequality for an operator $T$, we can define

$$
\mathcal{F}=\left\{(|T f|,|f|): f \in L_{c}^{\infty}\right\}
$$

alternatively, we can replace $L_{c}^{\infty}$ by $C_{c}^{\infty}, \mathcal{S}$, or any other appropriate dense subset of the spaces in question. To prove vector-valued inequalities for the operator $T$, we can use the pairs

$$
\left(\left(\sum_{k=1}^{M}\left|T f_{k}\right|^{r}\right)^{\frac{1}{r}},\left(\sum_{k=1}^{M}\left|f_{k}\right|^{r}\right)^{\frac{1}{r}}\right),
$$

where again the functions $f_{k}$ are taken from some appropriate dense subspace.

Below, to prove the vector-valued inequalities for the Hardy-Littlewood maximal operator and the fractional operator, we will use extrapolation; however, these results are more widely known and we will refer the reader to the literature. Here, we will first state two more recent versions of extrapolation in the scale of weighted spaces, and then prove two similar results which yield Lebesgue space inequalities.

Our first result is extrapolation in the scale of reverse Hölder weights. It was proved independently by Martell and Prisuelos [28] and in [3]. 
Theorem 3.1. Given $0<q_{0}<\infty$, suppose that for some $p_{0}, 0<p_{0} \leq q_{0}$ and all $w_{0} \in R H_{\left(\frac{q_{0}}{p_{0}}\right)^{\prime}}$

$$
\|f\|_{L^{p_{0}\left(w_{0}\right)}} \lesssim\|g\|_{L^{p_{0}\left(w_{0}\right)}}, \quad(f, g) \in \mathcal{F}
$$

Then for every $p, 0<p<q_{0}$, and $w \in R H_{\left(\frac{q_{0}}{p}\right)^{\prime}}$,

$$
\|f\|_{L^{p}(w)} \lesssim\|g\|_{L^{p}(w)}, \quad(f, g) \in \mathcal{F} .
$$

The second result is an off-diagonal, limited range extrapolation theorem recently proved in [8]. (We note in passing that this result contains essentially every other extrapolation theorem as a special case; see the discussion in the above paper for details.)

Theorem 3.2. Suppose $0<r<\infty$ and $0<p_{0}, q_{0}<\infty$ satisfy $0<p_{0} \leq r$ and $\frac{1}{q_{0}}-\frac{1}{p_{0}}+\frac{1}{r} \geq 0$, and $\mathcal{F}$ is a family of pairs of functions $(f, g)$. Further suppose that we have

$$
\left(\int_{\mathbf{R}^{n}}(f w)^{q_{0}} d x\right)^{\frac{1}{q_{0}}} \lesssim\left(\int_{\mathbf{R}^{n}}(g w)^{p_{0}} d x\right)^{\frac{1}{p_{0}}}
$$

for all $(f, g) \in \mathcal{F}$ and $\left.w \in R H_{\left(\frac{r}{p_{0}}\right)}\right)^{\prime}$. Then for every $0<p<r$ and $q$ such that $\frac{1}{p}-\frac{1}{q}=\frac{1}{p_{0}}-\frac{1}{q_{0}}$ and every weight $w$ such that $w^{p} \in R H_{\left(\frac{r}{p}\right)^{\prime}}$,

$$
\left(\int_{\mathbf{R}^{n}}(f w)^{q} d x\right)^{\frac{1}{q}} \lesssim\left(\int_{\mathbf{R}^{n}}(g w)^{p} d x\right)^{\frac{1}{p}}
$$

for all $(f, g) \in \mathcal{F}$.

We now prove two extrapolation theorems into the scale of variable Lebesgue spaces. In both proofs we use some well-known properties of the variable Lebesgue space norm; see [5] for details. We also use the properties of the Rubio de Francia iteration algorithm, which is an important tool in extrapolation theory. For a detailed discussion of this operator, see [9].

The first result is a generalization of Theorem 3.1.

Theorem 3.3. Given $0<p<q$, suppose that for all $w \in R H_{\left(\frac{q}{p}\right)^{\prime}}$,

$$
\|f\|_{L^{p}(w)} \lesssim\|g\|_{L^{p}(w)}, \quad(f, g) \in \mathcal{F} .
$$

Then for all $p(\cdot) \in \mathcal{P}_{0}$ such that $p<p_{-} \leq p_{+}<q$ and $p(\cdot) \in L H$,

$$
\|f\|_{L^{p(\cdot)}} \lesssim\|g\|_{L^{p(\cdot)}}, \quad(f, g) \in \mathcal{F} .
$$

Proof. For brevity, let $\tau=\frac{q}{p}$, and let $r(\cdot)=\frac{1}{\tau^{\prime}}\left(\frac{p(\cdot)}{p}\right)^{\prime}$. Since $p(\cdot) \in L H$, $r(\cdot) \in L H$. Further, we claim that $r_{-}>1$, which would imply that the maximal operator is bounded on $L^{r(\cdot)}$. To prove this, note that $r_{-}>1$ is equivalent to $\left[(p(\cdot) / p)^{\prime}\right]_{-}>\tau^{\prime}$, which in turn is equivalent to $[p(\cdot) / p]_{+}^{\prime}>\tau^{\prime}$, or $[p(\cdot) / p]_{+}<\tau$, which in turn is the same as our assumption that $p_{+}<q$.

Therefore, given non-negative $h$, we can define the Rubio de Francia iteration algorithm by

$$
\mathcal{R} h=\sum_{k=0}^{\infty} \frac{M^{k} h}{2^{k}\|M\|_{L^{r(\cdot)}}},
$$

where $M^{0} h=|h|$. This operator satisfies the following properties:

(1) $h \leq \mathcal{R} h$;

(2) $\|\mathcal{R} h\|_{r(\cdot)} \leq 2\|h\|_{r(\cdot)}$;

(3) $\mathcal{R} h \in A_{1}$ and $[\mathcal{R} h]_{A_{1}} \leq 2\|M\|_{L^{r(\cdot)}}$; 
(4) $\mathcal{R}\left(h^{\tau^{\prime}}\right) \frac{1}{\tau^{\prime}} \in A_{1} \cap R H_{\tau^{\prime}}$.

Now fix $(f, g) \in \mathcal{F}$ such that $\|f\|_{p(\cdot)}<\infty$. Then by duality,

$$
\|f\|_{p(\cdot)}^{p}=\left\|f^{p}\right\|_{p(\cdot) / p} \lesssim \int_{\mathbf{R}^{n}} f^{p} h d x
$$

where $h \geq 0, h \in L^{(p(\cdot) / p)^{\prime}}$ and $\|h\|_{(p(\cdot) / p)^{\prime}}=1$. Let $H=\mathcal{R}\left(h^{\tau^{\prime}}\right)^{\frac{1}{\tau^{\prime}}}$. By Hölder's inequality in the scale of variable Lebesgue spaces and the above properties,

$$
\begin{aligned}
\int f^{p} H d x & \lesssim\left\|f^{p}\right\|_{p(\cdot) / p}\|H\|_{(p(\cdot) / p)^{\prime}}=\|f\|_{p(\cdot)}^{p}\left\|\mathcal{R}\left(h^{\tau}\right)\right\|_{r(\cdot)} \\
& \lesssim\|f\|_{p(\cdot)}^{p}\left\|h^{\tau}\right\|_{r(\cdot)}=\|f\|_{p(\cdot)}^{p}<\infty
\end{aligned}
$$

Therefore, we can apply our hypothesis and repeat the above estimate with $g$ in place of $f$ to conclude that

$$
\begin{aligned}
\|f\|_{p(\cdot)}^{p} & \lesssim \int_{\mathbf{R}^{n}} f^{p} h d x \leq \int_{\mathbf{R}^{n}} f^{p} H d x \lesssim \int_{\mathbf{R}^{n}} g^{p} H d x \\
& \lesssim\left\|g^{p}\right\|_{p(\cdot) / p}\|H\|_{(p(\cdot) / p)^{\prime}} \lesssim\|g\|_{p(\cdot) \cdot}^{p}
\end{aligned}
$$

This completes the proof.

Our second result extends a special case of Theorem 3.2 to the variable Lebesgue spaces.

Theorem 3.4. Given $0<p<q<\infty$, suppose that for all $w^{p} \in R H_{\frac{q}{p}}$,

$$
\|f\|_{L^{q}\left(w^{q}\right)} \lesssim C\|g\|_{L^{p}\left(w^{p}\right)}, \quad(f, g) \in \mathcal{F} .
$$

Then for for all $p(\cdot) \in \mathcal{P}_{0}$ such that $p<p_{-} \leq p_{+}<\frac{1}{\frac{1}{p}-\frac{1}{q}}$ and $p(\cdot) \in L H$,

$$
\|f\|_{L^{q(\cdot)}} \lesssim\|g\|_{L^{p(\cdot)}}, \quad(f, g) \in \mathcal{F}
$$

where $q(\cdot)$ is defined by $\frac{1}{p(\cdot)}-\frac{1}{q(\cdot)}=\frac{1}{p}-\frac{1}{q}$.

Proof. The proof is very similar to the proof of Theorem 3.3, and we will omit some details. Define $r(\cdot)=\frac{p}{q}\left(\frac{p(\cdot)}{p}\right)^{\prime}$. Then $r(\cdot) \in L H$ and $r_{-}>1$ is equivalent to $\frac{1}{p}-\frac{1}{q}<\frac{1}{p_{+}}$. Hence, the maximal operator is bounded on $L^{r(\cdot)}$. Define the Rubio de Francia iteration algorithm for non-negative $h$ by

$$
\mathcal{R} h=\sum_{k=0}^{\infty} \frac{M^{k} h}{2^{k}\|M\|_{L^{r(\cdot)}}} .
$$

Then $\mathcal{R}$ satisfies

(1) $h \leq \mathcal{R} h$;

(2) $\|\mathcal{R} h\|_{r(\cdot)} \leq 2\|h\|_{r(\cdot)}$;

(3) $\mathcal{R} h \in A_{1}$ and $[\mathcal{R} h]_{A_{1}} \leq 2\|M\|_{L^{r(\cdot)}}$;

(4) $(\mathcal{R} h)^{\frac{p}{q}} \in A_{1} \cap R H_{\frac{q}{p}}$.

Now fix $(f, g) \in \mathcal{F}$ with $\|f\|_{L^{q(\cdot)}}<\infty$. Then

$$
\|f\|_{q(\cdot)}^{q}=\left\|f^{q}\right\|_{q(\cdot) / q} \lesssim \int_{\mathbf{R}^{n}} f^{q} h d x,
$$


where $h \geq 0$ and $\|h\|_{(q(\cdot) / q)^{\prime}}=1$. Let $H=(\mathcal{R} h)^{\frac{1}{q}}$, so that $h \leq H^{q}$, and $H^{p} \in R H_{\frac{q}{p}}$. Hence,

$$
\begin{aligned}
\int_{\mathbf{R}^{n}} f^{q} h d x & \leq \int_{\mathbf{R}^{n}} f^{q} H^{q} \lesssim\left(\int_{\mathbf{R}^{n}} g^{p} H^{p} d x\right)^{\frac{q}{p}} \\
& \lesssim\left\|g^{p}\right\|_{p(\cdot) / p}^{\frac{q}{p}}\left\|H^{p}\right\|_{(p(\cdot) / p)^{\prime}}^{\frac{q}{p}}=\|g\|_{p(\cdot)}^{q}\left\|H^{p}\right\|_{(p(\cdot) / p)^{\prime}}
\end{aligned}
$$

Moreover, since $\mathcal{R}$ was bounded on $L^{r(\cdot)}$,

$$
\left\|H^{p}\right\|_{(p(\cdot) / p)^{\prime}}=\left\|(\mathcal{R} h)^{\frac{p}{q}}\right\|_{(p(\cdot) / p)^{\prime}}=\|\mathcal{R} h\|_{\frac{p}{q}(p(\cdot) / p)^{\prime}}^{\frac{p}{q}} \lesssim\|h\|_{\frac{p}{q}(p(\cdot) / p)^{\prime}}^{\frac{p}{q}}
$$

We now claim that

$$
\frac{p}{q}\left(\frac{p(\cdot)}{p}\right)^{\prime}=\left(\frac{q(\cdot)}{q}\right)^{\prime} .
$$

To see this, observe that this is equivalent to

$$
\frac{1}{p\left(\frac{p(\cdot)}{p}\right)^{\prime}}=\frac{1}{q\left(\frac{q(\cdot)}{q}\right)^{\prime}}
$$

which is equivalent to our assumption that

$$
\frac{1}{p(\cdot)}-\frac{1}{q(\cdot)}=\frac{1}{p}-\frac{1}{q}
$$

Therefore, we have that

$$
\|h\|_{\frac{p}{q}(p(\cdot) / p)^{\prime}}^{\frac{p}{q}}=\|h\|_{(q(\cdot) / q)^{\prime}}^{\frac{p}{q}} \lesssim 1
$$

this completes the proof.

\section{Vector-valued inequalities}

We begin this section by stating four vector-valued inequalities for maximal operators on weighted and variable Lebesgue spaces. The first, for the Hardy-Littlewood maximal operator, was originally proved by Andersen and John [2]; here we want to stress that it is an immediate consequence via extrapolation [9, Section 3.8] of the scalar weighted norm inequalities for the maximal operator.

Lemma 4.1. Given $1<p, r<\infty$ and $w \in A_{p}$,

$$
\left\|\left(\sum_{k}\left(M g_{k}\right)^{r}\right)^{\frac{1}{r}}\right\|_{L^{p}(w)} \lesssim\left\|\left(\sum_{k}\left|g_{k}\right|^{r}\right)^{\frac{1}{r}}\right\|_{L^{p}(w)} .
$$

The second result on variable Lebesgue spaces, is also an immediate consequence of the scalar weighted norm inequalities and extrapolation [9, Theorem 4.25], [5, Corollary 5.34].

Lemma 4.2. Given $p(\cdot) \in \mathcal{P}_{0}$, such that $p(\cdot) \in L H$ and $1<p_{-} \leq p_{+}<\infty$, and $1<r<\infty$

$$
\left\|\left(\sum_{k}\left(M g_{k}\right)^{r}\right)^{\frac{1}{r}}\right\|_{L^{p(\cdot)}} \lesssim\left\|\left(\sum_{k}\left|g_{k}\right|^{r}\right)^{\frac{1}{r}}\right\|_{L^{p(\cdot)}} .
$$

The next two results are the analogues of the previous two for the fractional maximal operator. The first follows from the scalar, weighted inequalities for $M_{\alpha}$ and off-diagonal extrapolation [9, Theorem 3.23]. 
Lemma 4.3. Given $0<\alpha<n, 1<r<\infty$, and $1<p<\frac{n}{\alpha}$, define $q$ by $\frac{1}{p}-\frac{1}{q}=\frac{\alpha}{n}$. If $w \in A_{p, q}$, then

$$
\left\|\left(\sum_{k}\left(M_{\alpha} g_{k}\right)^{r}\right)^{\frac{1}{r}}\right\|_{L^{q}\left(w^{q}\right)} \lesssim\left\|\left(\sum_{k}\left|g_{k}\right|^{r}\right)^{\frac{1}{r}}\right\|_{L^{p}\left(w^{p}\right)} .
$$

The final inequality follows by off-diagonal extrapolation in the variable Lebesgue spaces [5, Theorem 5.28]. The vector-valued inequality is not explicitly proved there; however, it can be gotten by extrapolating starting with the weighted vector-valued inequality in [9, Theorem 3.23].

Lemma 4.4. Given $0<\alpha<n$ and $p(\cdot) \in \mathcal{P}_{0}$, suppose $1<p_{-} \leq p_{+}<\frac{n}{\alpha}$. Define $q(\cdot)$ by $\frac{1}{p(\cdot)}-\frac{1}{q(\cdot)}=\frac{\alpha}{n}$. Then

$$
\left\|\left(\sum_{k}\left(M_{\alpha} g_{k}\right)^{r}\right)^{\frac{1}{r}}\right\|_{L^{q(\cdot)}} \lesssim\left\|\left(\sum_{k}\left|g_{k}\right|^{r}\right)^{\frac{1}{r}}\right\|_{L^{p(\cdot)}} .
$$

Remark 4.5. In applying these vector-valued inequalities, we will use two generalizations. Rather than give these as corollaries, we instead describe the underlying ideas for adapting the above results. First, since maximal operators are positive homogeneous, we can, for example, replace $\left(M g_{k}\right)^{r}$ by $\lambda_{k}\left(M g_{k}\right)^{r}=\left(M\left(\lambda_{k}^{\frac{1}{r}} g_{k}\right)\right)^{r}$ on the left-hand side and $g_{k}^{r}$ by $\lambda_{k} g_{k}^{r}$ in the right-hand term.

Second, if we let $g_{k}=\chi_{Q_{k}}$ for some collection of cubes $Q_{k}$, then given $0<p<\infty$, $\tau>1$, and $w \in A_{\infty}$, there exists $r>1$ such that $w \in A_{r p}$, and so we have that

$$
\left\|\sum_{k} \chi_{\tau Q_{k}}\right\|_{L^{p}(w)} \lesssim\left\|\left(\sum_{k} M\left(\chi_{Q_{k}}\right)^{r}\right)^{\frac{1}{r}}\right\|_{L^{r p}(w)}^{r} \lesssim\left\|\sum_{k} \chi_{Q_{k}}\right\|_{L^{p}(w)} .
$$

We now turn to generalizations of the lemma of Grafakos and Kalton discussed in the introduction. First, though not actually necessary for the proof of our main results, we will show that by extrapolation we can easily prove a weighted version of their inequality.

Lemma 4.6. For $0<p \leq 1$, if $w \in R H_{\left(\frac{1}{p}\right)^{\prime}}$, then for all sequences of cubes $\left\{Q_{k}\right\}$ and non-negative functions $\left\{g_{k}\right\}$ such that $\operatorname{supp}\left(g_{k}\right) \subset Q_{k}$,

$$
\left\|\sum_{k} g_{k}\right\|_{L^{p}(w)} \lesssim\left\|\sum_{k}\left(f_{Q_{k}} g_{k} d y\right) \chi_{Q_{k}}\right\|_{L^{p}(w)} .
$$

Proof. We first prove this for $p=1$ and $w \in R H_{\infty}$. But in this case the result is trivial:

$$
\begin{aligned}
\left\|\sum_{k} g_{k}\right\|_{L^{1}(w)} & =\sum_{k} \int_{Q_{k}} g_{k} w d y \lesssim \sum_{k} \int_{Q_{k}} g_{k} d y f_{Q_{k}} w d y \\
& =\left\|\sum_{k}\left(f_{Q_{k}} g_{k} d y\right) \chi_{Q_{k}}\right\|_{L^{1}(w)} .
\end{aligned}
$$

The proof for $0<p<1$ and $w \in R H_{\left(\frac{1}{p}\right)^{\prime}}$ follows at once via reverse Hölder extrapolation, Theorem 3.1.

Below, we will use the following generalization of the lemma of Grafakos and Kalton, which lets us eliminate the hypothesis that $p \leq 1$ but replaces the $L^{1}$ averages by $L^{q}$ averages. 
Lemma 4.7. Fix $q>1$. If $0<p<q$ and $w \in R H_{\left(\frac{q}{p}\right)^{\prime}}$, then for all sequences of cubes $\left\{Q_{k}\right\}$ and non-negative functions $\left\{g_{k}\right\}$ such that $\operatorname{supp}\left(g_{k}\right) \subset Q_{k}$,

$$
\left\|\sum_{k} g_{k}\right\|_{L^{p}(w)} \lesssim\left\|\sum_{k}\left(f_{Q_{k}} g_{k}^{q} d y\right)^{\frac{1}{q}} \chi_{Q_{k}}\right\|_{L^{p}(w)} .
$$

Proof. We first prove this for $p=1$ and $w \in R H_{q^{\prime}}$. But in this case the result is trivial:

$$
\begin{aligned}
\left\|\sum_{k} g_{k}\right\|_{L^{1}(w)} & =\sum_{k} f_{Q_{k}} g_{k} w d y\left|Q_{k}\right| \leq \sum_{k}\left(f_{Q_{k}} g_{k}^{q} d y\right)^{\frac{1}{q}}\left(f_{Q_{k}} w^{q^{\prime}} d y\right)^{\frac{1}{q^{\prime}}}\left|Q_{k}\right| \\
& \lesssim \sum_{k}\left(f_{Q_{k}} g_{k}^{q} d y\right)^{\frac{1}{q}} w\left(Q_{k}\right)=\left\|\sum_{k}\left(f_{Q_{k}} g_{k}^{q} d y\right)^{\frac{1}{q}} \chi_{Q_{k}}\right\|_{L^{1}(w)} .
\end{aligned}
$$

The desired result for $0<p<q$ and $w \in R H_{\left(\frac{q}{p}\right)}$ follows at once from reverse Hölder extrapolation, Theorem 3.1.

Remark 4.8. For simplicity of statement and proof, we take $q>1$ in Lemma 4.7; but by Lemma 4.6 , if $0<p<1$, then we can take $q=1$.

We can now use extrapolation to extend the previous two results to the scale of variable Lebesgue spaces.

Lemma 4.9. Given $p(\cdot) \in \mathcal{P}_{0}$, suppose $0<p_{-} \leq p_{+}<1$ and $p(\cdot) \in L H$. Then for all sequences of cubes $\left\{Q_{k}\right\}$ and functions $\left\{g_{k}\right\}$ such that $\operatorname{supp}\left(g_{k}\right) \subset Q_{k}$,

$$
\left\|\sum_{k} g_{k}\right\|_{L^{p(\cdot)}} \lesssim\left\|\sum_{k}\left(f_{Q_{k}} g_{k} d y\right) \chi_{Q_{k}}\right\|_{L^{p(\cdot)}} .
$$

If we only assume that $p_{+}<\infty$, then for any $q$ such that $p_{+}<q<\infty$,

$$
\left\|\sum_{k} g_{k}\right\|_{L^{p(\cdot)}} \lesssim\left\|\sum_{k}\left(f_{Q_{k}} g_{k}^{q} d y\right)^{\frac{1}{q}} \chi_{Q_{k}}\right\|_{L^{p(\cdot)}} .
$$

Proof. The first inequality follows from Lemma 4.6 and Theorem 3.3 with $q=1$ and any $p$ such that $0<p<p_{-}$. The second inequality follows from Lemma 4.7 and Theorem 3.3 with $p_{+}<q<\infty$ and $0<p<p_{-}$.

The following off-diagonal inequality plays a role in the proof of Hardy space estimates for the fractional integral operator. It was first proved by Strömberg and Wheeden [39]; here we again give an elementary proof using extrapolation.

Lemma 4.10. Suppose $0<\alpha<n, 0<p<\frac{n}{\alpha}$, and $\frac{1}{q}=\frac{1}{p}-\frac{\alpha}{n}$. If $w^{p} \in R H_{\frac{q}{p}}$, then for any countable collection of cubes $\left\{Q_{k}\right\}$ and $\lambda_{k}>0$,

$$
\left\|\sum_{k} \lambda_{k}\left|Q_{k}\right|^{\frac{\alpha}{n}} \chi_{Q_{k}}\right\|_{L^{q}\left(w^{q}\right)} \lesssim\left\|\sum_{k} \lambda_{k} \chi_{Q_{k}}\right\|_{L^{p}\left(w^{p}\right)} .
$$

Proof. We will use Theorem 3.2 with $r=\frac{n}{\alpha}, p_{0}=1$, and $q_{0}=\frac{n}{n-\alpha}=\left(\frac{n}{\alpha}\right)^{\prime}$. We will show the estimate

$$
\left\|\sum_{k} \lambda_{k}\left|Q_{k}\right|^{\frac{\alpha}{n}} \chi_{Q_{k}}\right\|_{L^{\frac{n}{n-\alpha}\left(w^{\frac{n}{n-\alpha}}\right)}} \lesssim\left\|\sum_{k} \lambda_{k} \chi_{Q_{k}}\right\|_{L^{1}(w)}
$$


holds for all $w \in R H_{\frac{n}{n-\alpha}}$, all sequence of positive numbers $\left\{\lambda_{k}\right\}$, and sequences of cubes $\left\{Q_{k}\right\}$. If we assume (4.2) for the moment, then by the Theorem 3.2 we have that for all $0<p<\frac{n}{\alpha}, q$ satisfying $\frac{1}{p}-\frac{1}{q}=\frac{\alpha}{n}$, and $w$ such that $w^{p} \in R H_{\left(\frac{n / \alpha}{p}\right)^{\prime}}=$ $R H_{\frac{n}{n-\alpha p}}=R H_{\frac{q}{p}}$

$$
\left\|\sum_{k} \lambda_{k}\left|Q_{k}\right|^{\frac{\alpha}{n}} \chi_{Q_{k}}\right\|_{L^{q}\left(w^{q}\right)} \lesssim\left\|\sum_{k} \lambda_{k} \chi_{k}\right\|_{L^{p}\left(w^{p}\right)},
$$

which is the desired result.

To prove $(4.2)$, let $w \in R H_{\frac{n}{n-\alpha}}, u=w^{\frac{n}{n-\alpha}}$, and fix $g \geq 0$ in $L^{\left(\frac{n}{n-\alpha}\right)^{\prime}}\left(w^{\frac{n}{n-\alpha}}\right)=$ $L^{\frac{n}{\alpha}}(u)$. By duality, it will suffice to estimate the integral

$$
\int_{\mathbf{R}^{n}}\left(\sum_{k} \lambda_{k}\left|Q_{k}\right|^{\frac{\alpha}{n}} \chi_{Q_{k}}\right) g w^{\frac{n}{n-\alpha}} d x=\sum_{k} \lambda_{k}\left|Q_{k}\right|^{\frac{\alpha}{n}} \int_{Q_{k}} g u d x .
$$

But then we have that

$$
\begin{aligned}
\sum_{k} \lambda_{k}\left|Q_{k}\right|^{\frac{\alpha}{n}} \int_{Q_{k}} g u d x & \leq \sum_{k} \lambda_{k}\left|Q_{k}\right|^{\frac{\alpha}{n}}\left(\int_{Q_{k}} g^{\frac{n}{\alpha}} u d x\right)^{\frac{\alpha}{n}} u\left(Q_{k}\right)^{1-\frac{\alpha}{n}} \\
& \leq\|g\|_{L^{\frac{n}{\alpha}}(u)} \sum_{k} \lambda_{k}\left|Q_{k}\right|^{\frac{\alpha}{n}}\left(\int_{Q_{k}} w^{\frac{n}{n-\alpha}} d x\right)^{1-\frac{\alpha}{n}} \\
& \leq C\|g\|_{L^{\frac{n}{\alpha}}(u)} \sum_{k} \lambda_{k}\left|Q_{k}\right|^{\frac{\alpha}{n}} \int_{Q_{k}} w d x\left|Q_{k}\right|^{-\frac{\alpha}{n}} \\
& =C\|g\|_{L^{\frac{n}{\alpha}(u)}} \sum_{k} \lambda_{k} \int_{Q_{k}} w d x \\
& =C\|g\|_{L^{\frac{n}{\alpha}}(u)} \int_{\mathbf{R}^{n}}\left(\sum_{k} \lambda_{k} \chi_{Q_{k}}\right) w d x
\end{aligned}
$$

This completes the proof.

Our final estimate extends Lemma 4.10 to the variable Lebesgue spaces. It was first proved by Sawano [34]; however, it follows immediately from Lemma 4.10 by extrapolation, Theorem 3.4.

Lemma 4.11. Given $0<\alpha<n$, suppose $p(\cdot) \in \mathcal{P}_{0}$ is such that $p(\cdot) \in L H$ and $0<p_{-} \leq p_{+}<\frac{n}{\alpha}$. Define $q(\cdot)$ by $\frac{1}{p(\cdot)}-\frac{1}{q(\cdot)}=\frac{\alpha}{n}$. Then for any countable collection of cubes $\left\{Q_{k}\right\}$ and $\lambda_{k}>0$,

$$
\left\|\sum_{k} \lambda_{k}\left|Q_{k}\right|^{\frac{\alpha}{n}} \chi_{Q_{k}}\right\|_{q(\cdot)} \lesssim\left\|\sum_{k} \lambda_{k} \chi_{Q_{k}}\right\|_{p(\cdot)}
$$

\section{Singular integral operators}

In this section we prove Theorems 1.1 and 1.3. For the proof we need two lemmas; the essential ideas in their proofs are well-known (see, for instance, [37]) but to get the versions we need--which will be applicable to both singular integrals and fractional integrals-we give their short proofs. Throughout this section and Sections 6 and 7 below, let $\phi \in C_{c}^{\infty}$ be a fixed function supported in $B(0,1)$ with $\int \phi d x=1$. 
Lemma 5.1. Fix $N \geq 0$ and $0 \leq \alpha<n$. Let $K$ be a distribution such that $|\hat{K}(\xi)| \lesssim|\xi|^{-\alpha}$. Suppose further that away from the origin $K$ agrees with a function in $C^{N+1}$, and for all multi-indices $\beta$ such that $|\beta| \leq N+1$,

$$
\left|\partial^{\beta} K(x)\right| \leq \frac{B_{0}}{|x|^{n-\alpha+|\beta|}} .
$$

Define $K^{t}=\phi_{t} * K$. Then $K^{t}$ is a smooth function that satisfies

$$
\left|\partial^{\beta} K^{t}(x)\right| \leq \frac{B_{1}}{|x|^{n-\alpha+|\beta|}} .
$$

The constant $B_{1}$ is independent of $t$.

Proof. Fix $t>0$. Since $\phi$ is supported in the unit ball,

$$
K^{t}(x)=\int_{B(0, t)} \phi_{t}(y) K(x-y) d y .
$$

Suppose first that $|x|>2 t$. Then on $B(0, t)$ both functions and their derivatives are continuous and bounded, and so we can take the derivative inside the integral to get

$$
\begin{aligned}
\left|\partial^{\beta} K^{t}(x)\right| & \leq \int_{B(0, t)} t^{-n}|\phi(y / t)|\left|\partial^{\beta} K(x-y)\right| d y \\
& \lesssim t^{-n} \int_{B(0, t)}|x-y|^{-n+\alpha-|\beta|} d y \lesssim|x|^{-n+\alpha-|\beta|} .
\end{aligned}
$$

If $|x| \leq 2 t$, then by the inverse Fourier transform

$$
\partial^{\beta} K^{t}(x) \approx \int_{\mathbf{R}^{n}} e^{-2 \pi i x \cdot \xi} \xi^{\beta} \hat{K}(\xi) \hat{\phi}(t \xi) d \xi
$$

and so

$$
\begin{aligned}
\left|\partial^{\beta} K^{t}(x)\right| & \lesssim t^{\alpha-|\beta|} \int_{\mathbf{R}^{n}}|t \xi|^{-\alpha+|\beta|}|\hat{\phi}(t \xi)| d \xi \\
& \lesssim t^{-n+\alpha-|\beta|} \int_{\mathbf{R}^{n}}|u|^{-\alpha+|\beta|}|\hat{\phi}(u)| d u \lesssim|x|^{-n+\alpha-|\beta|} .
\end{aligned}
$$

The final integral converges since $\alpha<n$ and since $\hat{\phi}$ is a Schwartz function.

Lemma 5.2. Let $N, \alpha$ and $K$ be as in Lemma 5.1 and define the operator $T$ by $T f=K * f$. Let $a$ be any $(N, \infty)$ atom with $\operatorname{supp}(a) \subset Q$. Then for all $x \in\left(Q^{*}\right)^{c}$,

$$
M_{\phi}(T a)(x) \lesssim M_{\alpha_{\tau}}\left(\chi_{Q}\right)(x)^{\tau},
$$

where $\tau=\frac{n+N+1}{n}$ and $\alpha_{\tau}=\alpha / \tau$.

Proof. Fix $x \in\left(Q^{*}\right)^{c}$ and $t>0$. Then it will suffice to show that

$$
\left|\phi_{t} * T a(x)\right| \lesssim M_{\alpha_{\tau}}\left(\chi_{Q}\right)(x)^{\tau},
$$

with a constant independent of $x$ and $t$. Define $K^{t}=\phi_{t} * K$ as before. Since

$$
T a(x)=\int_{Q} K(x-y) a(y) d y
$$

and this integral converges absolutely, by taking the Fourier transform we see that $\phi_{t} * T a(x)=K^{t} * a(x)$. Let $P_{N}$ be the Taylor polynomial of degree $N$ of the function 
$y \rightarrow K^{t}(x-y)$ centered at $c_{Q}$. Then

$$
P_{N}(y)=\sum_{|\beta| \leq N} \frac{\partial^{\beta} K^{t}\left(x-c_{Q}\right)}{\beta !}\left(y-c_{Q}\right)^{\beta}
$$

so by the moment condition on $a$,

$$
\int_{Q} P_{N}(y) a(y) d y=0
$$

Moreover, the remainder $R_{N}(y)$ satisfies

$$
R_{N}(y)=K^{t}(x-y)-P_{N}(y)=\sum_{|\beta|=N+1} R_{\beta}(y)\left(y-c_{Q}\right)^{\beta}
$$

where

$$
R_{\beta}(y)=\int_{0}^{1}(1-s)^{N} \partial^{\beta} K^{t}\left(x-c_{Q}-s\left(y-c_{Q}\right)\right) d s .
$$

Therefore, by Lemma 5.1 we have that

$$
\begin{aligned}
\left|K^{t} * a(x)\right| & \leq \int_{Q}\left|K^{t}(x-y)-P_{N}(y)\right||a(y)| d y \\
& \lesssim \frac{1}{\left|x-c_{Q}\right|^{n-\alpha+N+1}} \int_{Q}\left|y-c_{Q}\right|^{N+1} d y \lesssim \frac{\ell(Q)^{n+N+1}}{\left|x-c_{Q}\right|^{n-\alpha+N+1}} .
\end{aligned}
$$

To complete the proof, note that for $x \in\left(Q^{*}\right)^{c}$, if $P$ is the smallest cube containing $x$ and $Q$, then

$$
M_{\alpha_{\tau}}\left(\chi_{Q}\right)(x)^{\tau} \approx\left[|P|^{\frac{\alpha_{\tau}}{n}} f_{P} \chi_{Q} d y\right]^{\tau} \approx\left[\frac{\ell(Q)^{n}}{\left|x-c_{Q}\right|^{n-\alpha_{\tau}}}\right]^{\tau}=\frac{\ell(Q)^{n+N+1}}{\left|x-c_{Q}\right|^{n-\alpha+N+1}} .
$$

If we combine these estimates we get the desired inequality.

Proof of Theorem 1.1. By the finite atomic decomposition, Proposition 2.1, it will suffice to fix a finite sum of $(N, \infty)$ atoms,

$$
f=\sum_{i=1}^{M} \lambda_{i} a_{i}
$$

with $\operatorname{supp}\left(a_{i}\right) \subset Q_{i}$ and $c_{i}=c_{Q_{i}}$, and prove that

$$
\left\|M_{\phi} T f\right\|_{L^{p}(w)} \lesssim\left\|\sum_{i=1}^{M} \lambda_{i} \chi_{Q_{i}}\right\|_{L^{p}(w)}
$$

By the linearity of $T$ and the sublinearity of $M_{\phi}$,

$$
\left\|M_{\phi} T f\right\|_{L^{p}(w)} \leq\left\|\sum_{i=1}^{M} \lambda_{i} M_{\phi} T\left(a_{i}\right) \chi_{Q_{i}^{*}}\right\|_{L^{p}(w)}+\left\|\sum_{i=1}^{M} \lambda_{i} M_{\phi} T\left(a_{i}\right) \chi_{\left(Q_{i}^{*}\right)^{c}}\right\|_{L^{p}(w)}=I_{1}+I_{2} .
$$

To estimate $I_{1}$, we apply Lemma 4.7. Since $w \in A_{\infty}, w \in R H_{s}$ for some $s>1$. Fix $q>\max (p, 1)$ such that $\left(\frac{q}{p}\right)^{\prime} \leq s$. Then $w \in R H_{\left(\frac{q}{p}\right)^{\prime}}$, and so by Lemma 4.7 and 
the fact that $T$ and $M_{\phi}$ are bounded on $L^{q}$,

$$
\begin{aligned}
I_{1} & \lesssim\left\|\sum_{i=1}^{M} \lambda_{i}\left(f_{Q_{i}} M_{\phi} T\left(a_{i}\right)^{q} d x\right)^{\frac{1}{q}} \chi_{Q_{i}^{*}}\right\|_{L^{p}(w)} \lesssim\left\|\sum_{i=1}^{M} \lambda_{i}\left(f_{Q_{i}}\left|a_{i}\right|^{q} d x\right)^{\frac{1}{q}} \chi_{Q_{i}^{*}}\right\|_{L^{p}(w)} \\
& \lesssim\left\|\sum_{i=1}^{M} \lambda_{i} \chi_{Q_{i}^{*}}\right\|_{L^{p}(w)} \lesssim\left\|\sum_{i=1}^{M} \lambda_{i} \chi_{Q_{i}}\right\|_{L^{p}(w)} ;
\end{aligned}
$$

the last inequality follows by Lemma 4.1 and Remark 4.5.

To estimate $I_{2}$, first note that by our assumption on $N$,

$$
N+1>n\left(\frac{r_{w}}{p}-1\right)
$$

or equivalently,

$$
p \tau=p\left(\frac{n+N+1}{n}\right)>r_{w} .
$$

Therefore, $w \in A_{p \tau}$, and so by Lemma 5.2 and Lemma 4.1,

$$
\begin{aligned}
I_{2} & \lesssim\left\|\sum_{i=1}^{M} \lambda_{i} M\left(\chi_{Q_{i}}\right)^{\tau}\right\|_{L^{p}(w)}=\left\|\left(\sum_{i=1}^{M} \lambda_{i} M\left(\chi_{Q_{i}}\right)^{\tau}\right)^{\frac{1}{\tau}}\right\|_{L^{p \tau}(w)}^{\tau} \\
& \lesssim\left\|\left(\sum_{i=1}^{M} \lambda_{i} \chi_{Q_{i}}\right)^{\frac{1}{\tau}}\right\|_{L^{p \tau}(w)}^{\tau}=\left\|\sum_{i=1}^{M} \lambda_{i} \chi_{Q_{i}}\right\|_{L^{p \tau}(w)} \cdot
\end{aligned}
$$

This completes the proof.

Proof of Theorem 1.3. The proof of this result is nearly identical to the above proof. By Proposition 2.4 we may again consider finite sums of atoms. We decompose as before into $I_{1}$ and $I_{2}$. To estimate $I_{1}$ we fix $q>\max \left(p_{+}, 1\right)$ and apply Lemma 4.9. We then use Lemma 4.2 and argue as in Remark 4.5. To estimate $I_{2}$, we note that $p_{-} \tau>1$, and so we can again apply Lemma 4.2.

\section{Fractional integral operators}

In this section we prove Theorems 1.5 and 1.7. The proof of Theorem 1.5 is very similar to the proof of Theorem 1.1 and so we will omit those details that are the same and concentrate on the differences. And again, the proof of Theorem 1.7 is a straightforward variation of the the proof of Theorem 1.5.

Proof of Theorem 1.5. We need to show that if $f$ is a finite sum of $(N, \infty)$ atoms,

$$
f=\sum_{i=1}^{M} \lambda_{i} a_{i}
$$

where the exact value of $N$ will be chosen below, then

$$
\left\|M_{\phi} I_{\alpha} f\right\|_{L^{q}\left(w^{q}\right)} \lesssim\left\|\sum_{i=1}^{M} \lambda_{i} \chi_{Q_{i}}\right\|_{L^{p}\left(w^{p}\right)} .
$$

As before we dominate the left-hand side by the sume of two terms:

$$
\left\|\sum_{i=1}^{M} \lambda_{i} M_{\phi} I_{\alpha}\left(a_{i}\right) \chi_{Q_{i}^{*}}\right\|_{L^{q}\left(w^{q}\right)}+\left\|\sum_{i=1}^{M} \lambda_{i} M_{\phi} I_{\alpha}\left(a_{i}\right) \chi_{\left(Q_{i}^{*}\right)^{c}}\right\|_{L^{q}\left(w^{q}\right)}=J_{1}+J_{2} .
$$


To estimate $J_{1}$, fix $q_{0}>\max \left(q, \frac{n}{n-\alpha}\right)$ and define $p_{0}>1$ by $\frac{1}{p_{0}}-\frac{1}{q_{0}}=\frac{\alpha}{n}$. Since $w^{p} \in R H_{\frac{q}{p}}, w^{q} \in A_{\infty}$, so arguing as before we may assume that $q_{0}$ is such that $w^{q} \in$ $R H_{\left(\frac{q_{0}}{p}\right)^{\prime}}$. Then by Lemma 4.7 , and since $M_{\phi}$ is bounded on $L^{q_{0}}$ and $I_{\alpha}: L^{p_{0}} \rightarrow L^{q_{0}}$,

$$
\begin{aligned}
J_{1} & \lesssim\left\|\sum_{i=1}^{M} \lambda_{i}\left(f_{Q_{i}} M_{\phi} I_{\alpha}\left(a_{i}\right)^{q_{0}} d x\right)^{\frac{1}{q_{0}}} \chi_{Q_{i}^{*}}\right\|_{L^{q}\left(w^{q}\right)} \\
& \lesssim\left\|\sum_{i=1}^{M} \lambda_{i}\left|Q_{i}\right|^{\frac{\alpha}{n}}\left(f_{Q_{i}}\left|a_{i}\right|^{p_{0}} d x\right)^{\frac{1}{p_{0}}} \chi_{Q_{i}^{*}}\right\|_{L^{q}\left(w^{q}\right)} \lesssim\left\|\sum_{i=1}^{M} \lambda_{i}\left|Q_{i}^{*}\right|^{\frac{\alpha}{n}} \chi_{Q_{i}^{*}}\right\|_{L^{q}\left(w^{q}\right)} .
\end{aligned}
$$

By Lemma 4.10 , since $w^{p} \in A_{\infty}$, by Lemma 4.1 we can continue this estimate, getting

$$
J_{1} \lesssim\left\|\sum_{i=1}^{M} \lambda_{i}\left|Q_{i}^{*}\right|^{\frac{\alpha}{n}} \chi_{Q_{i}^{*}}\right\|_{L^{q}\left(w^{q}\right)} \lesssim\left\|\sum_{i=1}^{M} \lambda_{i} \chi_{Q_{i}^{*}}\right\|_{L^{p}\left(w^{p}\right)} \lesssim\left\|\sum_{i=1}^{M} \lambda_{i} \chi_{Q_{i}}\right\|_{L^{p}\left(w^{p}\right)} .
$$

To estimate $J_{2}$ we will apply Lemma 5.2 , but first we need to fix $N$. For $I_{\alpha}$, our kernel is $K(x)=|x|^{\alpha-n}$, and so the desired estimates on the derivative of $K$ hold for all $N>0$. We now fix $N$ as follows: since $w^{q} \in A_{\infty}$, choose $N$ so that

$$
\left(\frac{n-\alpha+N+1}{n}\right) q>r_{w^{q}}
$$

As before, let $\tau=\frac{n+N+1}{n}$. Then, since $\frac{1}{\tau p}-\frac{1}{\tau q}=\frac{\alpha}{\tau n}$, we have that

$$
1+\frac{\tau q}{(\tau p)^{\prime}}=\tau q\left(1-\frac{\alpha}{\tau n}\right)=\left(\frac{n-\alpha+N+1}{n}\right) q .
$$

Hence, if we let $v=w^{\frac{1}{\tau}}$, we have that $v^{\tau q}=w^{q} \in A_{1+\frac{\tau q}{(\tau p)}}$. Equivalently, we have that $v \in A_{\tau p, \tau q}$. Therefore, by Lemma 5.2 and by Lemma 4.3 applied to the fractional maximal operator $M_{\alpha_{\tau}}$,

$$
\begin{aligned}
J_{2} & \lesssim\left\|\left(\sum_{i=1}^{M} \lambda_{i} M_{\alpha_{\tau}}\left(\chi_{Q_{i}}\right)^{\tau}\right)^{\frac{1}{\tau}}\right\|_{L^{q \tau}\left(v^{\tau q}\right)}^{\tau} \\
& \lesssim\left\|\left(\sum_{i=1}^{M} \lambda_{i} \chi_{Q_{i}}\right)^{\frac{1}{\tau}}\right\|_{L^{p \tau}\left(v^{\tau p}\right)}^{\tau}=\left\|\sum_{i=1}^{M} \lambda_{i} \chi_{Q_{i}}\right\|_{L^{p}\left(w^{p}\right)} .
\end{aligned}
$$

This completes the proof.

Proof of Theorem 1.7. The proof is essentially the same as the proof of Theorem 1.5. To estimate $J_{1}$ we use Lemma 4.9 with $q_{0}>\max \left(q_{+}, \frac{n}{n-\alpha}\right)$, and Lemma 4.11. To estimate $J_{2}$ we choose $N$ so large that $p_{-} \tau>1$ so that we can apply Lemma 4.4 to $M_{\alpha_{\tau}}$ acting from $L^{\tau p(\cdot)}$ to $L^{\tau q(\cdot)}$.

\section{Non-convolution operators}

The proofs of Theorems 1.9 and 1.11 for non-convolution Calderón-Zygmund operators are essentially identical to the proofs for convolution type singular integrals in Theorems 1.1 and 1.3. Fix such an operator $T$. Since it satisfies the standard kernel estimates (1.1) and (1.2), it is bounded on $L^{q}, 1<q<\infty$. Therefore, the estimate of the local piece is identical. To prove the estimate for the global piece, we need a maximal operator estimate for the action of $T$ on atoms, which is the substance of Lemma 7.2 below. The rest of the proof is identical. 
Remark 7.1. Our results can be generalized to a larger class of operators. An examination of the proof shows that to apply Lemma 4.7 and estimate the local piece, we need to assume that the operator $T$ is such that there exists $q>\max (p, 1)$ such that $w \in R H_{\left(\frac{q}{p}\right)^{\prime}}$ (in the weighted case) and $T$ satisfies $\|T a\|_{q} \lesssim|Q|^{\frac{1}{q}}$ (in either the weighted or variable exponent case). To estimate the global piece we again need that $T$ has a kernel $K$ representation such that (1.3) holds for all $|\beta| \leq L+1$ and $T$ satisfies (1.4) for all $|\beta| \leq L$, where $L$ is defined as before in Theorem 1.9 (for a weighted estimate) or Theorem 1.11 (for the variable exponent case). In particular, we do not assume that the operator is bounded on $L^{2}$ or that it satisfies the standard kernel estimates (1.1) and (1.2). We leave the details to the interested reader.

Lemma 7.2. Given $L \geq-1$, suppose $T$ is a Calderón-Zygmund operator associated with a kernel $K$ that satisfies (1.3) for all $|\beta|=L+1$, and suppose $T$ satisfies (1.4) for all $(L+1, \infty)$ atoms and $|\beta| \leq L$. (If $L=-1$ we disregard this condition.) Then given any $(L+1, \infty)$ atom $a, \operatorname{supp}(a) \subset Q$, then for all $x \in\left(Q^{* *}\right)^{c}$, and $\phi$ as defined in Section 5,

$$
M_{\phi}(T a)(x) \lesssim M\left(\chi_{Q}\right)(x)^{\frac{n+L+1}{n}} .
$$

Remark 7.3. As with Lemma 5.2, the ideas in the following proof are wellknown; for instance, in the multilinear case see [20, Lemma 3.3]. We include the details for completeness.

Proof. Fix $x \in\left(Q^{* *}\right)^{c}$; then to prove (7.1) it will suffice to prove that for all $t>0$,

$$
\left|\phi_{t} * T a(x)\right| \lesssim \frac{\ell(Q)^{n+L+1}}{|x-c|^{n+L+1}},
$$

where the implicit constant is independent of $t, x$ and $Q$. We will consider two cases: $0<t \leq \frac{|x-c|}{2}$ and $t>\frac{|x-c|}{2}$.

First, however, we will estimate the decay of $T a(y)$ when $y \in\left(Q^{*}\right)^{c}$. Let $N=L+1$ and let $c$ be the center of $Q$. By our assumption on the atom $a$ we have that $\int z^{\beta} a(z) d z=0$ for all $|\beta| \leq N$. Hence, we can apply Taylor's theorem with integral remainder to get

$$
\begin{aligned}
|T a(y)| & =\left|\int_{Q} K(y, z) a(z) d z\right|=\left|\int_{Q}\left[K(y, z)-\sum_{|\beta| \leq N-1} \frac{\partial_{z}^{\beta} K(y, c)}{\beta !}(z-c)^{\beta}\right] a(z) d z\right| \\
& =\left|\sum_{|\beta|=N} \frac{|\beta|}{\beta !} \int_{Q}\left(\int_{0}^{1}(1-\theta)^{N-1} \partial_{z}^{\beta} K\left(y, \zeta_{z, \theta}\right) d \theta\right)(z-c)^{\beta} a(z) d z\right|,
\end{aligned}
$$

where $\zeta_{z, \theta}=c+\theta(z-c)$. We again apply the vanishing moment condition of $a$ and (1.3) with $|\beta|=N$ to get

$$
\begin{aligned}
& =\left|\sum_{|\beta|=N} \frac{|\beta|}{\beta !} \int_{0}^{1}(1-\theta)^{N-1} \int\left(\partial_{z}^{\beta} K\left(y, \zeta_{z, \theta}\right)-\partial_{z}^{\beta} K(y, c)\right)(z-c)^{\beta} a(z) d z d \theta\right| \\
& \lesssim \int \frac{|z-c|^{\delta}}{|y-c|^{n+N+\delta}}|z-c|^{N}|a(z)| d z \lesssim \frac{\ell(Q)^{n+N+\delta}}{|y-c|^{n+N+\delta}} .
\end{aligned}
$$


We now consider the two cases given above. First, suppose $0<t \leq \frac{|x-c|}{2}$. Then we have that if $|x-y| \leq t$,

$$
|y-c| \geq|x-c|-|x-y| \geq|x-c|-t \geq \frac{1}{2}|x-c|,
$$

which, since $x \in\left(Q^{* *}\right)^{c}$, implies that $y \in\left(Q^{*}\right)^{c}$. Therefore, by (7.3) we have that

$$
\left|\phi_{t} * T a(x)\right| \leq \int_{|x-y| \leq t}\left|\phi_{t}(x-y)\right||T a(y)| d y \lesssim \frac{\ell(Q)^{n+N+\delta}}{|x-c|^{n+N+\delta}} \leq \frac{\ell(Q)^{n+L+1}}{|x-c|^{n+L+1}} .
$$

The last inequality holds since $\delta>0$ and $\ell(Q) \leq|x-c|$. This gives us (7.2) in the first case.

Now suppose that $t>\frac{|x-c|}{2}$. Then by the moment condition for Ta (1.4), and again by Taylor's theorem, we have that

$$
\begin{aligned}
\left|\phi_{t} * T a(x)\right| & =\left|\int_{Q} t^{-n} \phi\left(t^{-1}(x-y)\right) T a(y) d y\right| \\
& =\left|\int_{\mathbf{R}^{n}} t^{-n}\left[\phi\left(t^{-1}(x-y)\right)-\sum_{|\beta| \leq L} \frac{\partial^{\beta} \phi\left(\frac{x-c}{t}\right)}{\beta !}\left(\frac{c-y}{t}\right)^{\beta}\right] T a(y) d y\right| \\
& =\left|\int_{\mathbf{R}^{n}} \sum_{|\beta|=N} \frac{|\beta|}{t^{n} \beta !}\left(\int_{0}^{1}(1-\theta)^{L} \partial^{\beta} \phi\left(\frac{\zeta_{x, y, \theta}}{t}\right) d \theta\right)\left(\frac{c-y}{t}\right)^{\beta} T a(y) d y\right|,
\end{aligned}
$$

where $\zeta_{x, y, \theta}=x-c+\theta(c-y)$. If we replace the inner integral by the supremum of the integrand, we get

$$
\begin{aligned}
\left|\phi_{t} * T a(x)\right| & \lesssim \sum_{|\beta|=N} t^{-n-N} \int_{\mathbf{R}^{n}} \sup _{0 \leq \theta \leq 1}\left|\partial^{\beta} \phi\left(\frac{\zeta_{x, y, \theta}}{t}\right)\right||c-y|^{N}|T a(y)| d y \\
& \lesssim|x-c|^{-n-N} \int_{\mathbf{R}^{n}}|y-c|^{N}|T a(y)| d y .
\end{aligned}
$$

To estimate the final integral we split the domain. On $\left(Q^{*}\right)^{c}$ we use (7.3) to get

$$
\int_{\left(Q^{*}\right)^{c}}|y-c|^{N}|T a(y)| d y \lesssim \int_{\left(Q^{*}\right)^{c}} \frac{\ell(Q)^{n+N+\delta}}{|y-c|^{n+\delta}} d y \lesssim \ell(Q)^{n+N} .
$$

On the other hand, to estimate the integral on $Q^{*}$ we use the fact that $T$ is bounded on $L^{r}$ for $r>1$. Then by Hölder's inequality we have that

$$
\int_{Q^{*}}|y-c|^{N}|T a(y)| d y \lesssim \ell(Q)^{n / r^{\prime}+N}\|T a\|_{L^{r}} \lesssim \ell(Q)^{n+N}
$$

If we combine all of these estimates, we see that for $t>\frac{|x-c|}{2}$,

$$
\left|\phi_{t} * T a(x)\right| \lesssim \frac{\ell(Q)^{n+L+1}}{|x-c|^{n+L+1}}
$$

which give us (7.2) in this case. This completes the proof. 


\section{Extensions to other Banach function spaces}

In this section we conclude by briefly considering the extension of our approach to Hardy spaces defined with respect to other quasi-Banach function spaces. Our starting point is the observation that we were able to prove results for the variable Hardy spaces because we could prove vector-valued inequalities in the variable Lebesgue spaces in Section 4 via extrapolation from the corresponding weighted norm inequalities.

Therefore, to extend our results to other scales of spaces we need a theory of extrapolation. In [9], motivated by the extrapolation results to the scale of variable Lebesgue spaces in [6], the authors considered the general problem of extrapolating from weighted norm inequalities into quasi-Banach function spaces. Their approach was the following: given a quasi-Banach function space $X$, define a scale of spaces $X^{r}, 0<r<\infty$, where $f \in X^{r}$ if $|f|^{r} \in X$, and the "norm" on $X^{r}$ is given by

$$
\|f\|_{X^{r}}=\left\||f|^{r}\right\|_{X}^{\frac{1}{r}} \text {. }
$$

In order to use extrapolation to prove results in $X$, it is necessary to assume that there exists $r>1$ such that $X^{r}$ is a Banach function space, and that the Hardy-Littlewood maximal operator is bounded on the associate space $\left(X^{r}\right)^{\prime}$. (See [9, Remark 4.7].) Thus, for example, in the scale of variable Lebesgue spaces, given $p(\cdot) \in \mathcal{P}_{0}$, we would fix $0<p<p_{-}$and use the fact that if $p(\cdot) \in L H$, the maximal operator is bounded on the Banach function space $L^{(p(\cdot) / p)^{\prime}}$.

Given a quasi-Banach space $X$, we can define a Hardy space $H^{X}$ to be the set of all distributions $f$ such that $\mathcal{M}_{N_{0}} f \in X$, with quasi-norm $\|f\|_{H^{X}}=\left\|\mathcal{M}_{N_{0}} f\right\|_{X}$. The question is then whether we can prove that singular and fractional integrals are bounded on the spaces $H^{X}$. The proof would require two components. First, we would need the theory of extrapolation to prove the various vector-valued inequalities required. Second, we would need the basics of Hardy space theory: in particular, the equivalence of the various definitions of a Hardy space in terms of the radial and grand maximal operators, and the finite atomic decomposition.

We could, for instance, apply these ideas to the Hardy-Orlicz spaces introduced by Janson [24], and considered earlier in the case of analytic functions on the unit disk by Leśniewicz [26]. An atomic decomposition for these spaces was given by Viviani [40]. Another, more recent example of spaces that would be amenable to our approach are the Musielak-Orlicz Hardy spaces. These are based on the MusielakOrlicz spaces introduced in [30]; see [41] for a comprehensive treatment, including an atomic decomposition. (We note in passing that extrapolation into the scale of Musielak-Orlicz spaces was considered separately in [7].) Additional examples Hardy spaces where our approach might be applicable are given in [36].

\section{References}

[1] Alvarez, J., and M. Milman: $H^{p}$ continuity properties of Calderón-Zygmund-type operators. - J. Math. Anal. Appl. 118:1, 1986, 63-79.

[2] Andersen, K. F., and R. T. John: Weighted inequalities for vector-valued maximal functions and singular integrals. - Studia Math. 69:1, 1980/81, 19-31.

[3] Anderson, T., and D. Cruz-Uribe, and K. Moen: Extrapolation in the scale of generalized reverse Hölder weights. - Rev. Mat. Complut. 31:2, 2018, 263-286.

[4] Coifman, R., and Y. Meyer: Au delà des opérateurs pseudo-différentiels. - Astérisque 57, Soc. Math. France, Paris, 1978. 
[5] Cruz-Uribe, D., and A. Fiorenza: Variable Lebesgue spaces: Foundations and harmonic analysis. - Birkhäuser, Basel, 2013.

[6] Cruz-Uribe, D., A. Fiorenza, J. M. Martell, and C. PÉrez: The boundedness of classical operators on variable $L^{p}$ spaces. - Ann. Acad. Sci. Fenn. Math. 31:1, 239-264, 2006.

[7] Cruz-Uribe, D., and P. Hästö: - Extrapolation and interpolation in generalized Orlicz spaces. - Trans. Amer. Math. Soc. 370:6, 2018, 4323-4349.

[8] Cruz-Uribe, D., and J. M. Martell: Limited range multilinear extrapolation with applications to the bilinear Hilbert transform. - Math. Ann. 371:1-2, 2018, 615-653.

[9] Cruz-Uribe, D., J. M. Martell, and C. Pérez: Weights, extrapolation and the theory of Rubio de Francia. - Operator Theory Adv. Appl. 215, Birkhäuser/Springer Basel AG, Basel, 2011.

[10] Cruz-Uribe, D., K. Moen, and H. V. Nguyen: The boundedness of multilinear CalderónZygmund operators on weighted and variable Hardy spaces. - Publ. Mat. (to appear).

[11] Cruz-Uribe, D., and L.-A. Wang: Variable Hardy spaces. - Indiana Univ. Math. J. 63:2, 2014, 447-493.

[12] Diening, L., P. Harjulehto, P. Hästö, and M. RŮŽičKa: Lebesgue and Sobolev spaces with variable exponents. - Lecture Notes in Math. 2017, Springer, Heidelberg, 2011.

[13] Duonndikoetxea, J.: Fourier analysis. - Grad. Stud. Math. 29, Amer. Math. Soc., Providence, RI, 2001.

[14] Fefferman, C., and E. M. Stein: Some maximal inequalities. - Amer. J. Math. 93, 107-115, 1971.

[15] García-Cuerva, J.: Weighted $H^{p}$ spaces. - Dissertationes Math. (Rozprawy Mat.) 162, 1979.

[16] García-Cuerva, J., and J. L. Rubio de Francia: Weighted norm inequalities and related topics. - North-Holland Mathematics Studies 116, North-Holland Publishing Co., Amsterdam, 1985.

[17] Gatto, A. E., C. E. Gutiérrez, and R. L. Wheeden: Fractional integrals on weighted $H^{p}$ spaces. - Trans. Amer. Math. Soc. 289:2, 1985, 575-589.

[18] Grafakos, L., and D. He: Weak Hardy spaces. - In: Some topics in harmonic analysis and applications, Adv. Lect. Math. (ALM) 34, Int. Press, Somerville, MA, 2016, 177-202.

[19] Grafakos, L., and N. Kalton: Multilinear Calderón-Zygmund operators on Hardy spaces. - Collect. Math. 52:2, 2001, 169-179.

[20] Grafakos, L., S. Nakamura, H. V. Nguyen, and Y. Sawano: Conditions for Boundedness into Hardy spaces. - Preprint, arXiv:1702.02229v1, 2017.

[21] Grafakos, L., S. Nakamura, H. V. Nguyen, and Y. Sawano: Multiplier conditions for boundedness into Hardy spaces. - Ann. Inst. Fourier (Grenoble) (to appear).

[22] Hart, J., and G. Lu: Hardy space estimates for Littlewood-Paley-Stein square functions and Calderón-Zygmund operators. - J. Fourier Anal. Appl. 22:1, 2016, 159-186.

[23] Hart, J., and L. Oliveira: Hardy space estimates for limited ranges of Muckenhoupt weights. - Adv. Math. 313, 2017, 803-838.

[24] JAnson, S.: Generalizations of Lipschitz spaces and an application to Hardy spaces and bounded mean oscillation. - Duke Math. J. 47:4, 1980, 959-982.

[25] Krantz, S. G.: Fractional integration on Hardy spaces. - Studia Math. 73:2, 1982, 87-94.

[26] Leśniewicz, R.: On Hardy-Orlicz spaces. I. - Bull. Acad. Polon. Sci. Sér. Sci. Math. Astronom. Phys. 14, 1966, 145-150.

[27] Lu, G., and Y. ZHU: Bounds of singular integrals on weighted Hardy spaces and discrete Littlewood-Paley analysis. - J. Geom. Anal. 22:3, 2012, 666-684. 
[28] Martell, J. M., and C. Prisuelos-Arribas: Weighted Hardy spaces associated with elliptic operators. Part I: Weighted norm inequalities for conical square functions. - Trans. Amer. Math. Soc. 369:6, 2017, 4193-4233.

[29] Meda, S., P. Sjögren, and M. Vallarino: On the $H^{1}-L^{1}$ boundedness of operators. - Proc. Amer. Math. Soc. 136:8, 2008, 2921-2931.

[30] Musielak, J.: Orlicz spaces and modular spaces. - Lecture Notes in Math. 1034, SpringerVerlag, Berlin, 1983.

[31] NAKAI, E., and Y. SAWANO: Hardy spaces with variable exponents and generalized Campanato spaces. - J. Funct. Anal. 262:9, 2012, 3665-3748.

[32] Rocha, P., and M. Urciuolo: Fractional type integral operators on variable Hardy spaces. - Acta Math. Hungar. 143:2, 2014, 502-514.

[33] Ruiz Blasco, F. J., and J. L. Torrea Hernández: Weighted and vector-valued inequalities for potential operators. - Trans. Amer. Math. Soc. 295:1, 1986, 213-232.

[34] Sawano, Y.: Atomic decompositions of Hardy spaces with variable exponents and its application to bounded linear operators. - Integral Equations Operator Theory 77:1, 2013, 123-148.

[35] Sawano, Y.: Theory of Besov spaces. - Dev. Math. 56, Springer, Singapore, 2018.

[36] Sawano, Y., and K.-P. Ho, D. YAng, and S. YAnG: Hardy spaces for ball quasi-Banach function spaces. - Dissertationes Math. 525, 2017.

[37] Stein, E. M.: Harmonic analysis: real-variable methods, orthogonality, and oscillatory integrals. - Princeton Math. Ser. 43, Princeton Univ. Press, 1993.

[38] Strömberg, J.-O., and A. Torchinsky: Weighted Hardy spaces. - Lecture Notes in Math. 1381, Springer-Verlag, Berlin, 1989.

[39] Strömberg, J.-O., and R. L. Wheeden: Fractional integrals on weighted $H^{p}$ and $L^{p}$ spaces. - Trans. Amer. Math. Soc. 287:1, 1985, 293-321.

[40] Viviani, B. E.: An atomic decomposition of the predual of $\operatorname{BMO}(\rho)$. - Rev. Mat. Iberoamericana 3:3-4, 1987, 401-425.

[41] YAng, D., Y. LiAng, and L. D. KY: Real-variable theory of Musielak-Orlicz Hardy spaces. Lecture Notes in Math. 2182, Springer, Cham, 2017.

Received 5 February 2019 • Accepted 8 March 2019 